\title{
Opt Art
}

\author{
Robert Bosch \\ Oberlin College, Oberlin $\mathrm{OH} 44074$, USA \\ DominoArtwork.com, Oberlin OH 44074, USA
}

\begin{abstract}
Optimization deals with finding the best way to complete a task - creating a schedule for a tournament, matching professors with courses, constructing an itinerary for a traveling salesman. It has been applied successfully to such a great number of diverse disciplines that one could argue that it can be put to good use in every imaginable field. In this talk, we will showcase its amazing utility by describing some applications in the area of art: portraits constructed out of complete sets of dominoes (via integer programming) mosaics comprised of abstract geometric tiles (via integer programming and various heuristics), and continuous line drawings (via the "solution" of large-scale instances of the traveling salesman problem).
\end{abstract}
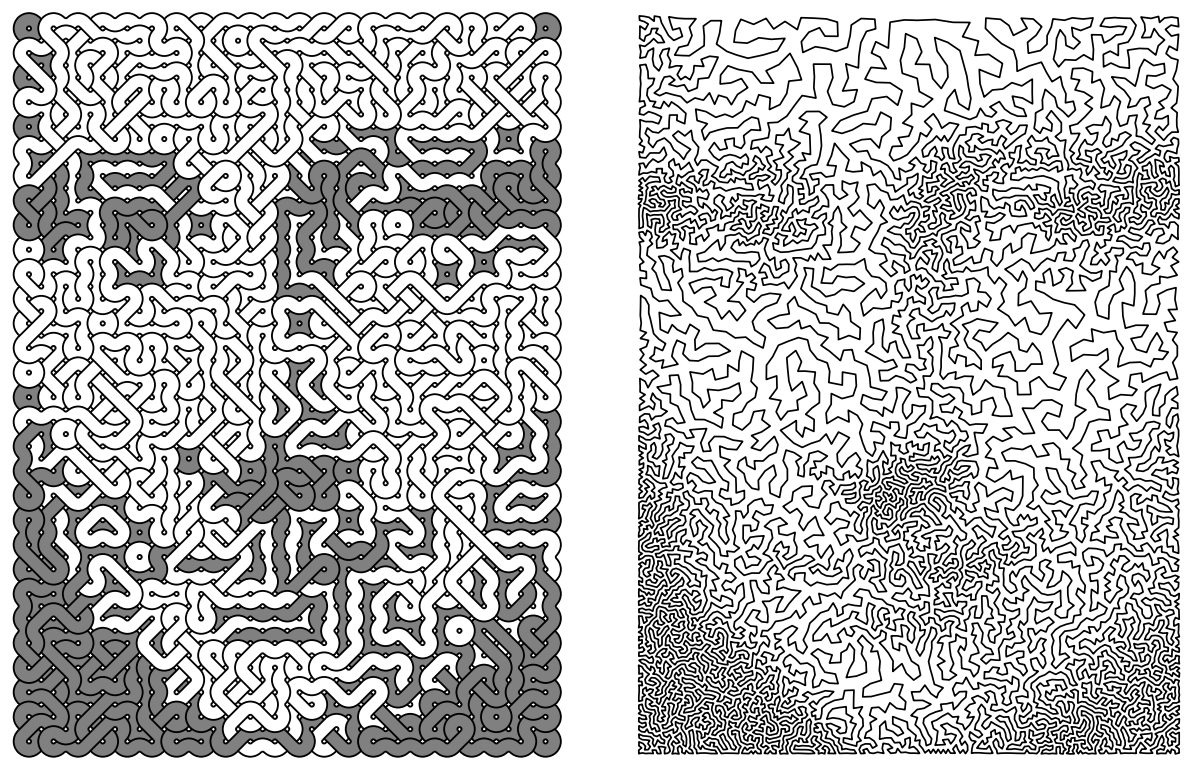

Fig. 1. Examples of Opt Art 